\title{
Gestión del tiempo y compromiso académico en estudiantes de psicología de una universidad privada de Juliaca
}

\section{Time management and academic commitment in psychology students from a private university in Juliaca}

\section{Daniel Eduardo Cahuana Cuti ${ }^{1}$}

\begin{abstract}
RESUMEN
Objetivo: determinar si existe relación significativa entre la gestión del tiempoy el compromiso académico en estudiantes de psicología de una universidad privada. Metodología: diseño no experimental, de corte transversal, tipo correlacional y de enfoque cuantitativo. La muestra estuvo constituida por 217 estudiantes, los cuales fueron seleccionados a través del muestreo probabilístico estratificado donde se segmento en 5 grupos de población de estudiantes de psicología de las edades de 18 años a más, tomando en cuenta varones y damas que están matriculados en el ciclo estudiantil 2021-1. Los instrumentos utilizados fueron el Time Management Behavior Scale (TMBS), este instrumento alcanza un indicie de confianza de ,867 y una validez en un $V=, 99$. Respecto al instrumento Utrecht Work Engagement Student (UWES), este instrumento alcanza un índice de confianza de ,917 y una validez en un $\mathrm{V}=, 99$; estos instrumentos indican un grado de validez y confiabilidad que catalogan que ambos instrumentos miden lo que deberían medir y de forma consistente. Resultados: la gestión del tiempo y el compromiso académico se correlacionan de manera positiva y estadísticamente significativa ( $r=$ ,335, $p=, 000)$. Así mismo existe correlación en la dimensión vigor (Rho=,373; $p=, 000)$, dedicación $(\mathrm{Rho}=, 231 ; \mathrm{p}=, 000)$ y Absorción ( $\mathrm{Rho}=, 287 ; \mathrm{p}=, 000)$, siendo altamente significativas. Conclusión: a mayor gestión del tiempo mayor compromiso académico.
\end{abstract}

Palabras clave: Gestión del tiempo; compromiso académico; estudiantes universitarios.

\begin{abstract}
Objective: to determine if there is a significant relationship between time management and academic commitment in psychology students from a private university. Methodology: non-experimental design, cross-sectional, correlational type and quantitative approach. The sample consisted of 217 students, who were selected through stratified probability sampling where they were divided into 5 population groups of psychology students aged 18 years and over, taking into account men and women who are enrolled in the student cycle 2021-1. The instruments used were the Time Management Behavior Scale (TMBS), this instrument reaches a confidence index of .867 and a validity of $V=.99$. Regarding the Utrecht Work Engagement Student (UWES) instrument, this instrument achieves a confidence index of .917 and a validity of $V=.99$; These instruments indicate a degree of validity and reliability that shows that both instruments measure what they should measure consistently. Results: time management and academic commitment are positively and statistically significantly correlated $(r=.335, p=.000)$. Likewise, there is a correlation in the vigor dimension (Rho $=.373 ; p=.000)$, dedication (Rho =. 231; $p=.000)$ and Absorption (Rho $=.287 ; p=.000$ ), being highly significant. Conclusion: the greater the time management, the greater the academic commitment.
\end{abstract}

Keywords: Time management; academic commitment; University students.

${ }^{1}$ Universidad Peruana Unión, Juliaca, Perú.

Orcid ID: 0000-0002-5655-7364 


\section{INTRODUCCIÓN}

En la actualidad la emergencia sanitara (covid-19), ha obligado a la sociedad académica a explorar novedosas maneras de enseñar y aprender, incluyendo la educación a distancia en línea, esta situación resulto difícil tanto para el estudiante y el docente, teniendo que afrontar problemas emocionales, físicos y económicos, al mismo tiempo contribuir a frenar la propagación de esta enfermedad, siendo el principal problema de salud pública, declarada así por la Organización de las Naciones Unidas (ONU, 2020).

La nueva modalidad de aprendizaje favorece que los estudiantes puedan acceder a los materiales de estudio y realicen sus actividades académicas en cualquier momento y cualquier lugar, de una forma flexible y dinámica. El uso de aulas virtuales requiere autorregulación por parte de los estudiantes, para que sean capaces de alcanzar el proceso de aprendizaje (Gros, 2018). Uno de los factores de autorregulación es la gestión del tiempo. Si un estudiante no logra planificar y organizar bien su tiempo de estudio y dejar todo a último momento es probable que se presenten problemas de desmotivación, apatía para completar los trabajos o actividades, bajo rendimiento e incluso que abandone los estudios. (Van Laer \& Elen, 2017)

Una investigación realizada por el Banco Mundial a finales del 2018, reveló que los estudiantes de 25 a 29 años de edad no finalizaron sus estudios, sea porque no continuaron estudiando o desertaron; también se estimó que el $30 \%$ de estudiantes que empieza una carrera universitaria abandonara el sistema de educación superior al final del primer año (Ferreira et al, 2018). Según el Minedu (2020) afirma que la tasa de deserción universitaria en el Perú alcanzo un $18,6 \%$ a diferencia del 2019 que fue un $12 \%$.

En el Perú el $27 \%$ de los ingresantes a una universidad privada abandonaran sus estudios en el primer año, algunas causas de la deserción universitaria son: bajo rendimiento académico, dudas vocacionales, problemas económicos y problemas emocionales (Analytics, 2017).

El adaptarse a la vida universitaria demanda autonomía, seguridad y confianza en toma de decisiones, e implica adaptarse a nuevas exigencias académicas. Durante el proceso formativo varios estudiantes universitarios no consiguen desarrollar habilidades $y$ competencias necesarias que les permita sobrellevar situaciones de estrés y experimentan sensaciones de no poder consigo mismo, actitud de fracaso y sentirse incapaz de realizar tareas académicas (Palacio, Caballero, Gonzales, Gravini, \& Contreras, 2012).

Para tener un buen rendimiento académico dependerá mucho de una adecuada gestión y organización del tiempo. Saber planificar, tener las adecuadas herramientas, estar motivado y tener confianza. La gestión adecuada del estudio permite lograr buenos resultados y evitar momentos de agobio (Marchena et al. 2017). Otro factor importante es el compromiso académico, vinculado con la dedicación a los estudios, organización de tareas asignadas, exposiciones y trabajos grupales y la disposición que tiene el estudiante de participar en actividades académicas de su escuela profesional y universidad, con el objetivo de desarrollarse académicamente (Baños, 2020). Muchos estudiantes presentan problemas para gestionar su tiempo y tener compromiso académico ya que no utilizan herramientas para organizar sus actividades, ocasionando una escasa dedicación a los estudios y como consecuencia un bajo rendimiento o deserción académica.

Baños (2020) en su investigación titulada la gestión del tiempo y compromiso académico en estudiantes de psicología de la universidad privada Norbert Wiener. La investigación estuvo enfocada en conocer la relación entre gestión del tiempo y compromiso académico con un método cuantitativo, de tipo correlacional descriptivo. Utilizando 2 instrumentos los cuales fueron la escala de comportamiento de gestión del tiempo y la escala de compromiso académico. El estudio estuvo conformado por 157 damas y 52 varones, los resultados hallados revelan que ambas variables se correlacionan de manera positiva y estadísticamente significativa $(\mathrm{Rho}=$ .304, $p=001)$, la dimensión vigor $(\mathrm{Rho}=.378, p=$ 001) y absorción (Rho = .286, $p=001)$. También, se demostró que no existe correlación con la dedicación $(R h o=.109, p=117)$. En conclusión, los estudiantes universitarios de psicología demuestran niveles moderados de gestión del tiempo $(52 \%)$ y compromiso académico (55\%).

Así también Cotrina y Sanchez (2020) en su investigación titulada; gestión del tiempo como 
factor predictor del rendimiento académico en adolescentes que estudian en la institución educativa "Dos de mayo" Cajamarca, dicha investigación es de tipo cuantitativo y de alcance correlacional-transversal. Se utilizo el instrumento Time Management Behavior Scale TMBS, instrumento que permitió medir la gestión del tiempo, así mismo para medir el rendimiento académico se utilizó los calificativos de los estudiantes correspondientes al primer trimestre. Tal estudio estuvo conformado por 209 estudiantes, donde se halló una correlación directa entre gestión del tiempo y rendimiento académico $(R=0,664)$ y concluyendo que la gestión del tiempo si es un factor predictor del rendimiento académico, en una proporción de $44,1 \%$, en los estudiantes del segundo al quinto grado de educación.

Ordoñez (2020) en su investigación titulada resiliencia y compromiso académico en estudiantes de una universidad de Quevedo. La investigación tiene enfoque cuantitativo y diseño no experimental, transversal y correlacional, se utilizó la "Escala de resiliencia" (ER-14) dicho instrumento permitió medir la resiliencia y para la medición del compromiso académico se utilizó la escala Engagementen el estudio en universitarios UWES-S 17, la cual estuvo conformada por 9 ítems. Dicho estudio estuvo conformado por 155 estudiantes de la Universidad de Quevedo, cuyas edades oscilaban entre los 18 a 24 años de edad, seleccionadas mediante muestreo no probabilístico intencional. Los resultados mostraron que existe relación altamente significativa (Rho= 643; $p<.01$ ) entre resiliencia y compromiso académico en estudiantes de la Universidad de Quevedo. Además, se identificó que existe relación significativa $(p=.001)$ entre la dimensión competencia personal y las dimensiones vigor con $(\mathrm{Rho}=.532)$, dedicación (Rho=.558), y absorción (Rho=.533), lo cual significa que, a mayor competencia personal, mayores puntajes entre Vigor, dedicación y absorción. Esto significa que la muestra de estudio presenta autoconfianza, decisión, independencia y perseverancia.

La gestión del tiempo es la expresión conductual de una serie de procesos internos vinculados con la autorregulación para que el individuo tenga probabilidades de alcanzar sus metas, y seguir sus objetivos en la medida que emplee tiempo en realizarlas (Kearns \& Gardiner, 2007). Según Krause y Coates (2008) señalan que la capacidad de gestionar el tiempo promueve el éxito académico y que la constancia genera un hábito de un nuevo comportamiento, lo cual contribuye que el estudiante genere estrategias de aprendizaje.

Macan, Shahani, Dipboye y Phillips (1990) argumentan que tener una inadecuada gestión del tiempo y no darle prioridad a las actividades académicas o laborales son las principales fuentes de fatiga, tensión, agotamiento, y disminución del rendimiento académico. Así mismo, propone que las conductas para gestionar el tiempo no se hallan relacionadas con problemas psicológicos o somáticos, sino con la percepción del tiempo y las conductas de administrar el tiempo.

Macan et al (1990) plantea un modelo de gestión del tiempo donde se enfatiza la importancia de establecer metas y prioridades, mecanismos para cumplir tareas y preferencias por la desorganización. La escala establece 4 dimensiones. Establecer objetos y prioridades: evalúa la disposición del estudiante para establecer, objetivos y prioridades para la realización de actividades académicas. Esto lleva a la organización de su tiempo y lograr sus metas propuestas. Mecánica de gestión del tiempo planificación y programación: esta dimensión se encuentra asociada a la gestión eficaz del tiempo como emplear métodos de organización del tiempo como horarios, registro de actividades pendientes, utilización de agendas y otros. Preferencias de desorganización: vinculada como el estudiante aborda sus tareas y actividades y mostrando conductas ineficaces de gestión del tiempo tales como la improvisación, trabajo desordenado o la procrastinación. Percepción de control sobre el tiempo: esta dimensión aborda como el estudiante percibe su tiempo y como lo utiliza usando herramientas para una buena gestión del tiempo: establece objetivos y prioridades.

El compromiso académico forma parte de la actuación que tendrá el alumno dentro del entorno académico, de esta forma el compromiso, se refiere a la vinculación, la implicación, la pasión, el entusiasmo, el esfuerzo y la energía respecto a lo que el individuo hace (Casuso, 2011). El compromiso dentro del entorno académico, recibirá el nombre de "engagement" académico, cuya traducción más literal al español es de compromiso académico según Hinrichs, Ortiz, y Pérez (2016). Asimismo, este nuevo criterio se aproxima al entorno educativo, el cual hace 
alusión a la sensación de confort que muestra el alumno frente a los retos académicos (Parra \& Pérez, 2010).

El engagement académico se apoya en la teoría social cognitiva de la autoeficacia de Albert Bandura, Siendo Shaufeli et al (2002) quienes elaboran la escala de Utrecht de Engagement Academic bajo los lineamientos teóricos de Bandura. Por su parte señala que las creencias de la autoeficacia se pueden aprender a incrementar o disminuir a través de 4 rutas principales. La primera ruta son las experiencias previas de autoeficacia: se refiere cuando la persona logra con frecuencia el éxito en sus actividades se incrementa la autoeficacia sin embargo cuando hay constantes fracasos se incrementa las expectativas negativas de autoeficacia. La segunda es experiencia vicaria: se produce cuando el observador percibe que personas a su alrededor realizan con éxitos ciertas actividades, entonces el observador cree que también puede tener éxito. La tercera ruta es la persuasión verbal: es la fuente de autoeficacia, solo necesitan un poco de confianza para poner su mayor esfuerzo y así conseguir el éxito. La última ruta es el estado fisiológico del individuo: posee indicadores de cansancio, dolores y pueden ser apreciados por los individuos como signos de incompetencia, el estado de ánimo es un papel muy importante.

Al principió los estudios de compromiso fueron en el área laboral, posteriormente este constructo fue ampliado e investigado en el área académica (Schaufeli \& Bakker, 2003). Dentro de este estudio definen al compromiso como un elemento de motivación intrínseca que perciben los estudiantes, siendo esta motivación constante. Asimismo, señalan que existen 3 dimensiones las cuales son: vigor, dedicación y absorción. La primera dimensión es vigor: vinculado con la energía que tienen los estudiantes mientras estudian, siendo constante también cuando hay dificultades. La segunda dimensión es dedicación: asociado con el entusiasmo, la importancia y el orgullo relacionado con la carrera de estudio profesional. Finalmente, la dimensión absorción: asociada con la atención plena del estudiante, se parecía como el estado de felicidad, connotación de placer y motivación en las actividades académicas.

Por lo anteriormente expuesto se tiene como objetivo determinar la relación de la gestión del tiempo y compromiso académico en estudiantes de psicología de una universidad privada de Juliaca, 2021. Los objetivos específicos procuran determinar la relación de la gestión del tiempo y las dimensiones de compromiso académico que son: vigor, dedicación y absorción.

\section{METODOLOGÍA}

En el presente estudio se optó por un diseño no experimental de corte transversal; debido que no hay manipulaciones deliberadas de variables y los datos se recolectan en un solo momento, es de tipo correlacional, la cual tiene la finalidad de estudiar la relación entre las variables. Es decir, consiste en proporcionar la descripción de los hechos tal como se dan y correlacionar la variable $A$ con la variable $B$ y subvariables. Es de enfoque cuantitativo porque el instrumento usado proporcionara resultados numéricos las cuales serán procesados bajo esa índole (Hernández, Fernández, \& Baptista, 2014).

\section{Participantes}

Respecto a la población según Hernández, Fernández y Baptista (2014), consideran que una población es un conjunto finito o infinito de personas $u$ objetos que tienen características comunes, siendo así la población de investigación los estudiantes de psicología de una universidad privada de Juliaca. La población de investigación estuvo constituida por 979 estudiantes universitarios que oscilan entre las edades de 18 años a más. La muestra estuvo constituida por 217 estudiantes, los cuales fueron seleccionados a través del muestreo probabilístico estratificado. Donde se segmentará en 5 grupos de población de estudiantes de psicología tomando en cuenta varones y damas que están matriculados en el ciclo estudiantil 2021-1 siendo alumnos regulares y excluyendo a los alumnos irregulares. 
Tabla 1

Muestra de participantes

\begin{tabular}{llll} 
Estrato por grupo & Año académico de estudiantes de psicología & Total, de población & Muestra \\
\hline 1 & Primer Año & 389 & 86 \\
2 & Segundo Año & 257 & 57 \\
3 & Tercer Año & 162 & 36 \\
4 & Cuarto Año & 94 & 21 \\
5 & Quinto Año & 77 & 17 \\
Total & & $\mathrm{N}=979$ & $\mathrm{n}=217$ \\
\hline
\end{tabular}

\section{Instrumentos}

Para la recolección de datos se utilizó el instrumento Time Management Behavior Scale (TMBS) cuya autoría fue por Macan et al (1990), adaptado al español por García y Pérez (2012), llevando por título la Escala de Comportamiento de gestión del tiempo. Posee 34 ítems con una escala Likert con cinco opciones de respuesta que van del 1 al 5 tales como: $1=$ nunca, $2=$ pocas veces, $3=$ algunas veces, 4=habitualmente, 5=siempre. El instrumento consta de 4 dimensiones: Establecimiento de objetivos y prioridades, herramientas para la gestión del tiempo, percepción de control del tiempo, preferencias por la desorganización. Estas dimensiones están destinadas a evaluar la gestión del tiempo académico en alumnos de educación superior. Los valores de validez fueron hallados por análisis factorial exploratorio, en donde se evidencia que el $42,93 \%$ de la varianza representa al constructo y su grado de confiabilidad hallada por consistencia interna de coeficiente de alfa de por dimensiones, tales como: dimensión 1 obtuvo un alfa de 0.84 , dimensión 2 obtuvo 0.79 , dimensión 3 obtuvo 0.71 y la dimensión 4 obtuvo 0.72 siendo valores aceptados.

Así mismo, se utilizó el instrumento Utrecht Work Engagement Student (UWES) cuya autoría fue por Schaufeli y Bakker (2003) y validado en el contexto peruano por Moreno (2019), que lleva por título la Escala de Utrecht de Engagement Académico. Posee 17 ítems con una escala Likert con seis opciones de respuesta que van del 0 al 6 tales como: 0=nunca, 1=casi nunca, 2=algunas veces, 3=regularmente, 4= bastantes veces, $5=$ casi siempre, $6=$ siempre. El instrumento consta de 3 dimensiones: Vigor, dedicación y absorción. Destinados a identificar el compromiso académico y el funcionamiento óptimo de los estudiantes. Los valores de validez y confiabilidad por alfa de Cronbach se estimaron en un $95 \%$ de confianza y sus dimensiones, tales como: dedicación que obtuvo un estimado de .912, asimismo en la escala de vigor y absorción la confiabilidad fue de .793 y .772 calificado como aceptable.

En cuanto a la validez de contenido se obtuvo mediante el coeficiente de $\mathrm{V}$ de Aiken donde Escurra (1988) menciona que los valores mayores o iguales a 0.80 se consideran válidos para la prueba. De esta manera fueron sometidos a evaluación mediante juicio de 5 expertos en el área, valorando la claridad, congruencia, contexto, dominio de constructo.

En la tabla 2 se muestra la validez de $\mathrm{V}$ de Aiken, donde se obtuvo un puntaje en el constructo total de 0,99 este resultado indica que el instrumento es válido para su uso en investigación. Respecto a las dimensiones, establecimiento de objetivos y prioridades alcanza un nivel de $V=1$ este resultado indica que la dimensión es válida en cuanto a su contenido. Respecto a la dimensión herramientas para la gestión del tiempo esta obtiene un $\mathrm{V}=0.99$ este resultado indicaría que la dimensión es válida en cuanto a su contenido. Respecto a la dimensión percepción del control del tiempo alcanza un nivel de $V=0.99$ este resultado indicaría que la dimensión es válida en cuanto a su contenido. Respecto a la dimensión preferencias por la desorganización alcanza un nivel de $\mathrm{V}=0.99$ este resultado indicaría que la dimensión es válida en cuanto a su contenido. 
Tabla 2

Validez de contenido para el instrumento Time Management Behavior Scale

\begin{tabular}{lllll} 
Dimensiones & Claridad & Congruencia & Contexto & $\begin{array}{c}\text { Dominio de } \\
\text { Constructo }\end{array}$ \\
Establecimiento de objetivos y prioridades & 1 & 1 & 1 & 1 \\
Herramientas para la gestión del tiempo & 0.98 & 1 & 0.98 & 1 \\
Percepción del control del tiempo & 0.96 & 1 & 1 & 1 \\
Preferencias por la desorganización & 1 & 0.98 & 1 & 0.99 \\
Total & 0.985 & 0.994 & 0.995 & 0.99 \\
\hline
\end{tabular}

En la tabla 3 se muestra la validez de $V$ de Aiken, donde se obtuvo un puntaje en el constructo total de 0,99 este resultado indica que el instrumento es válido para su uso en investigación. Respecto a las dimensiones, vigor alcanza un nivel de $\mathrm{V}=1$ este resultado indica que la dimensión es válida en cuanto a su contenido. Respecto a la dimensión dedicación esta obtiene un $\mathrm{V}=0.98$ este resultado indicaría que la dimensión es válida en cuanto a su contenido. Respecto a la dimensión absorción alcanza un nivel de $\mathrm{V}=1$ este resultado indicaría que la dimensión es válida en cuanto a su contenido.

Tabla 3

Validez de contenido para el instrumento Utrecht Work Engagement Student

\begin{tabular}{llllll} 
Dimensiones & Claridad & Congruencia & Contexto & $\begin{array}{l}\text { Dominio de } \\
\text { Constructo }\end{array}$ & V de aiken \\
\hline Vigor & 1 & 1 & 1 & 1 & 1 \\
Dedicación & 0.96 & 1 & 0.96 & 1 & 0.98 \\
Absorción & 1 & 1 & 1 & 1 & 1 \\
Total & 0.99 & 1 & 0.99 & 1 & 0.99 \\
\hline
\end{tabular}

En cuanto a la confiabilidad, se utilizó el Alpha de Cronbach: Un índice de consistencia interna con valores entre 0 y 1 . Utilizando el SPSS se obtuvo la fiabilidad de los instrumentos.

En la tabla 4 se muestra los resultados del alfa de Cronbach siendo el resultado de, 867 , lo cual indica que es recomendable su aplicación para este estudio; los resultados por dimensiones fueron ,838, para la dimensión establecimiento de objetivos y prioridades; ,868, para la dimensión herramientas para la gestión del tiempo; ,346, para la dimensión percepción del control del tiempo; ,617, para la dimensión preferencias por la desorganización.

Tabla 4

Confiabilidad del instrumento Time Management Behavior Scale

\begin{tabular}{lll} 
Dimensiones & Alpha & N elementos $^{\circ}$ \\
Establecimiento de objetivos y prioridades &, 838 & 10 \\
Herramientas para la gestión del tiempo &, 868 & 11 \\
Percepción del control del tiempo &, 346 & 5 \\
Preferencias por la desorganización &, 617 & 8 \\
General &, 867 & 34 \\
\hline
\end{tabular}

En la tabla 5 se muestra los resultados del alfa de Cronbach siendo el resultado de ,917, lo cual indica que es recomendable su aplicación para este estudio; los resultados por dimensiones fueron ,804, para la dimensión vigor; ,860, para la dimensión dedicación; ,765, para la dimensión absorción. 
Tabla 5

Confiabilidad del instrumento Utrecht Work Engagement Student

\begin{tabular}{lcc} 
Dimensiones & Alpha & $N^{\circ}$ elementos \\
\hline Vigor &, 804 & 6 \\
Dedicación &, 860 & 5 \\
Absorción &, 765 & 6 \\
General &, 917 & 17 \\
\hline
\end{tabular}

\section{Análisis de datos}

Después de recolectar la información mediante el google formulario, se utilizó el software SPSS en su versión 26 . Seguidamente se hizo la fiabilidad de los instrumentos luego se extrajo los estadísticos de frecuencia respecto a los datos sociodemográficos, de la misma manera se obtuvieron las tablas de frecuencia de personas que se sitúan en determinados niveles por cada variable de estudio. Para determinar el tipo de distribución de las variables, se hará uso de la prueba de Kolmogorov Smirnov; los resultados demuestran que las variables poseen una distribución no normal, luego se hizo la prueba de Rho de Sperman para determinar el índice de correlaciones entre las variables.

\section{RESULTADOS}

\section{Análisis descriptivo sociodemográfico}

En la tabla 6 se puede observar que la población estuvo conformada por 217 estudiantes. Respecto a la variable año académico, el $39,6 \%$ pertenece al primer; el $26,3 \%$ pertenece al segundo año; el $16,6 \%$ pertenece al tercer año; el 9,7\% pertenece al cuarto año; el 7,8\% pertenece al quinto año. En cuanto a la variable género, el $68,7 \%$ son damas y el $31,3 \%$ son varones. Por otra parte, en la variable edad fue de 18 años a más. Siguiendo con la variable condición laboral el $21,7 \%$ es dependiente; el 18,4 es independiente y el $59,9 \%$ aun no trabaja. Finalmente, en cuanto a la variable preferencias religiosas, $39.6 \%$ son adventistas; el $55,3 \%$ son de otras religiones y el $5,1 \%$ no es creyente.

Tabla 6

Análisis sociodemográficos de la población de estudio

\begin{tabular}{llll} 
& & Frecuencia & Porcentaje \\
Año Académico & 1 & 86 & $39,6 \%$ \\
& 2 & 57 & $26,3 \%$ \\
& 3 & 36 & $16,6 \%$ \\
Genero & 4 & 21 & $9,7 \%$ \\
& 5 & 17 & $7,8 \%$ \\
Edad & Femenino & 149 & $68,7 \%$ \\
Condición laboral & Masculino & 68 & $31,3 \%$ \\
& 18 años a más & & \\
Preferencia religiosa & Dependiente & 47 & $21,7 \%$ \\
& Independiente & 40 & $18,4 \%$ \\
& Aun no trabaja & 130 & $59,9 \%$ \\
& Adventista & 86 & $39,6 \%$ \\
& Otros & 120 & $55,3 \%$ \\
\hline
\end{tabular}

\section{Análisis descriptivo}

En la tabla 7 se muestra los niveles de gestión del tiempo en estudiantes de psicología de una universidad privada de Juliaca, donde se aprecia que el $73,3 \%$ de los estudiantes universitarios presentan un nivel de gestión del tiempo medio, el 14,7\% de los estudiantes universitarios presentan un nivel Alto y solo un $12,0 \%$ presentan un nivel de gestión del tiempo bajo. 
Tabla 7

Nivel de Gestión del tiempo en estudiantes universitarios de psicología de una universidad privada de Juliaca, 2021.

\begin{tabular}{|c|c|c|c|c|c|c|c|c|}
\hline & Bajo & & Medi & & Alto & & Total & \\
\hline & $f$ & $\%$ & $f$ & $\%$ & $f$ & $\%$ & $f$ & $\%$ \\
\hline $\begin{array}{l}\text { Nivel de Gestión del } \\
\text { tiempo }\end{array}$ & 26 & 12,0 & 159 & 73,3 & 33 & 14,7 & 217 & 100,0 \\
\hline
\end{tabular}

En la tabla 8 se muestra los niveles de compromiso académico en estudiantes de psicología de una universidad privada de Juliaca, donde se aprecia que el $68,7 \%$ de los estudiantes universitarios presentan un nivel de gestión del tiempo medio, el $16,6 \%$ de los estudiantes universitarios presentan un nivel Alto y solo un $14,7 \%$ presentan un nivel de gestión del tiempo bajo.

Tabla 8

Nivel de Compromiso académico en estudiantes de psicología de una universidad privada de Juliaca, 2021

\begin{tabular}{lllllllll} 
& \multicolumn{1}{c}{ Bajo } & \multicolumn{3}{c}{ Medio } & \multicolumn{3}{c}{ Alto } & \multicolumn{2}{c}{ Total } \\
\cline { 2 - 9 } & $\mathrm{f}$ & $\%$ & $\mathrm{f}$ & $\%$ & $\mathrm{f}$ & $\%$ & $\mathrm{f}$ & $\%$ \\
\hline $\begin{array}{l}\text { Nivel de Compromiso } \\
\text { académico }\end{array}$ & 32 & 14,7 & 149 & 68,7 & 36 & 16,6 & 217 & 100,0 \\
\hline
\end{tabular}

Prueba de normalidad

En la tabla 9, se observa las pruebas de normalidad para ambas variables, donde su nivel de sig. para la gestión del tiempo es 0,48 y para compromiso académico su nivel de sig. ,000. Entonces concluimos que provienen de una distribución no normal teniendo un $p<0.05$ para las dos variables. Estos datos demandan que las variables deben ser procesadas inferencialmente con estadísticos de correlación no paramétricos.

Tabla 9

Prueba de normalidad para variables gestión del tiempo y compromiso académico

\begin{tabular}{llll} 
& \multicolumn{2}{l}{ Prueba de Kolmogorov-Smirnov } \\
\cline { 2 - 4 } & Estadístico & $\mathrm{gl}$ & Sig. \\
\hline Gestión del tiempo &, 061 & 217 &, 048 \\
Compromiso académico & 0,94 & 217 &, 000 \\
\hline
\end{tabular}

\section{En relación con la hipótesis general}

En la tabla 10, se observa la correlación entre la variable gestión del tiempo y compromiso académico, donde se puede apreciar la intensidad de correlación es de ,335 que lo ubica en un nivel de intensidad medio-baja, con respecto al nivel de significancia apreciamos que es ,000 cuyo valor es menor del 0,05 entonces podemos decir que existe relación estadísticamente significativa. 
Tabla 10

Correlación de Rho de Sperman entre las variables Gestión del tiempo y Compromiso académico

\begin{tabular}{|c|c|c|c|c|}
\hline & & & $\begin{array}{l}\text { Total Gestión } \\
\text { del tiempo }\end{array}$ & $\begin{array}{l}\text { Total Compromiso } \\
\text { académico }\end{array}$ \\
\hline \multirow[t]{6}{*}{ Rho de Spearman } & Gestión del Tiempo & $\begin{array}{l}\text { Coeficiente de } \\
\text { correlación }\end{array}$ & 1,000 &, $335^{* *}$ \\
\hline & & Sig. (bilateral) & . & ,000 \\
\hline & & $\mathrm{N}$ & 217 & 217 \\
\hline & Compromiso Académico & $\begin{array}{l}\text { Coeficiente de } \\
\text { correlación }\end{array}$ &, $335^{\star *}$ & 1,000 \\
\hline & & Sig. (bilateral) &, 000 & . \\
\hline & & $\mathrm{N}$ & 217 & 217 \\
\hline
\end{tabular}

\section{En relación con las hipótesis especificas}

En la tabla 11 se pueden observar las correlaciones por Rho de Sperman para variables con distribución no normal que revelan que existe una correlación estadísticamente significativa. Así mismo se evidencia las correlaciones entre la variable gestión del tiempo y las dimensiones de la variable compromiso académico. Siendo la correlación en la dimensión vigor (Rho=,373; $p=, 000)$ cuya intensidad de correlación es medio-baja, existiendo una relación estadísticamente significativa. En la dimensión dedicación $(\mathrm{Rho}=, 231 ; \mathrm{p}=, 000)$ cuya intensidad de correlación es medio-baja, existiendo una relación estadísticamente significativa y en la dimensión Absorción ( $R h o=, 287 ; p=, 000)$ cuya intensidad de correlación es medio-baja, existiendo una relación estadísticamente significativa. Así mismo se evidencia las correlaciones entre la variable Compromiso académico y las dimensiones de la variable gestión del del tiempo. Siendo la correlación en la dimensión Establecimiento de objetivos y prioridades donde $(\mathrm{Rho}=, 423 ; \mathrm{p}=, 000)$ cuya intensidad de correlación es media, existiendo una relación estadísticamente significativa. En la dimensión Herramientas para la gestión del tiempo (Rho=,376; $p=, 000)$ cuya intensidad de correlación es medio-baja, existiendo una relación estadísticamente significativa. En la dimensión percepción del control del tiempo (Rho= -,020; $p=, 771)$ donde no existe relación estadísticamente significativa y en la dimensión preferencias por la desorganización (Rho= $-, 104 ; p=, 126)$ donde no existe relación estadísticamente significativa.

Tabla 11

Estadísticos de correlación entre la variable gestión del tiempo y compromiso académico con sus respectivas dimensiones

\begin{tabular}{lll} 
& \multicolumn{2}{l}{ Gestión del tiempo } \\
\cline { 2 - 2 } Compromiso académico & Rho & \\
Vigor &, $373^{*}$ &, 000 \\
Dedicación &, $231^{*}$ &, 000 \\
Absorción &, $287^{*}$ &, 000 \\
Gestión del tiempo & \\
Establecimiento de objetivos y prioridades &, $423^{*}$ &, 000 \\
Herramientas para la gestión del tiempo &, $376^{*}$ &, 000 \\
Percepción del control del tiempo &,$- 020^{*}$ &, 771 \\
Preferencias por la desorganización &,$- 104^{*}$ &, 126 \\
\hline
\end{tabular}

Nota: $\left(^{*}\right)$ Los coeficientes de correlación pueden ser interpretados a un 0,01 de confianza. 


\section{DISCUSIÓN}

El objetivo general de esta investigación, fue determinar la relación entre la gestión del tiempo y compromiso académico en estudiantes de psicología de una universidad privada de Juliaca, 2021. A partir del análisis estadístico permitió establecer la existencia de correlaciones positivas entre la variable gestión del tiempo con las dimensiones vigor, dedicación, absorción y la variable compromiso académico. Hallando entre nuestras dos variables de estudio un $(\mathrm{Rho}=, 335, \mathrm{p}=, 000)$, existiendo una relación estadísticamente significativa; estos resultados coinciden con lo reportado en la literatura científica; por ejemplo, con la investigación de Baños (2020) quien también abordo la gestión del tiempo y compromiso académico en estudiantes de psicología de una universidad privada en la ciudad de Lima, siendo sus resultados (Rho $=.304, p=001$ ), evidenciando asociaciones significativas entre ambas variables. Por otro lado, Cotrina y Sanchez (2020) en un estudio realizado en estudiantes de nivel secundario en la ciudad de Cajamarca, hallaron una correlación directa entre la gestión del tiempo y rendimiento académico la cual fue $(r=, 664$, $p=, 000$ ). En base a esto, se puede asumir que en la medida que los estudiantes asuman la responsabilidad de gestionar su tiempo tendrán mayor compromiso académico. Por lo tanto, uno de los factores de autorregulación es la gestión del tiempo. Si un estudiante no logra planificar y organizar bien su tiempo de estudio y dejar todo a último momento es probable que se presenten problemas de desmotivación, apatía para completar los trabajos o actividades, bajo rendimiento e incluso que abandone los estudios (Van Laer \& Elen, 2017).

Asimismo, en cuanto al primer objetivo específico, referido a determinar la relación entre la gestión del tiempo y la dimensión vigor, se encontró un $(R h o=, 373 ; p=, 000)$ cuya intensidad de correlación es medio-baja, existiendo una relación estadísticamente significativa. Estos resultados coinciden con lo reportado en la literatura científica; por ejemplo, con la investigación de Baños (2020) sus resultados evidenciaron asociaciones entre la gestión de tiempo y la dimensión vigor $(\mathrm{Rho}=.378, \mathrm{p}=001)$ donde se puede indicar que a mayor gestión del tiempo mayor vigor en los estudiantes universitarios. En base a esto se puede asumir que en la medida que los estudiantes tengan una mejor gestión del tiempo, entonces mayor será la energía y esfuerzo que invierta en las actividades académicas y la persistencia para afrontar dificultades académicas.

Con respecto al segundo objetivo específico, referidoadeterminarlarelaciónentrelagestión del tiempo y la dimensión dedicación de compromiso académico, se encontró un $(\mathrm{Rho}=, 231 ; \mathrm{p}=$ $, 000)$ cuya intensidad de correlación es mediobaja, existiendo una relación estadísticamente significativa. Asimismo, Ordoñez (2020) en su investigación encontró asociaciones entre la dimensión competencia personal y la dimensión dedicación de compromiso académico (Rho=.558; $p=, 001$ ) hallando que la población de estudio presenta decisión, independencia y perseverancia. En base a esto se puede asumir que en la medida que los estudiantes tengan una mejor gestión del tiempo, entonces mayor será el compromiso, motivación e interés hacia las actividades académicas.

Por otro parte el tercer objetivo específico, referido a determinar la relación entre la gestión del tiempo y la dimensión absorción se encontró un $(R h o=, 287 ; p=, 000)$, encontrando una correlación medio-baja, estadísticamente significativa. Estos resultados coinciden con los resultados de la investigación de Baños (2020) donde se evidencio asociaciones entre la gestión de tiempo y la dimensión absorción (Rho $=.286, p=001)$. donde se puede indicar que a mayor gestión del tiempo mayor absorción en los estudiantes universitarios. Por lo cual en la medida que los estudiantes tengan una mayor gestión del tiempo, entonces mayor será la disposición, concentración, disfrute y satisfacción en el desarrollo de las actividades académicas.

Asimismo, otro aspecto relevante de los resultados encontrados en los estudiantes universitarios de psicología, demuestra que el $73,3 \%$ presenta un nivel medio de gestión del tiempo, de igual forma con respecto al compromiso académico el $68,7 \%$ un nivel medio. Considerando que los estudiantes universitarios de psicología se han adaptado el uso de aulas virtuales ya que esto requiere autorregulación por parte de los estudiantes, para que sean capaces de alcanzar el proceso de aprendizaje (Gros, 2018). Asimismo, el compromiso académico forma parte de la actuación que tendrá el alumno dentro del entorno académico, de esta forma el compromiso, se refiere a la vinculación, la implicación, la pasión, el 
entusiasmo, el esfuerzo y la energía respecto a lo que el individuo hace (Casuso, 2011). Por tanto, la gestión adecuada del estudio permite lograr buenos resultados y evitar momentos de agobio (Marchena et al. 2017).

Finalmente, la literatura científica da cuenta sobre la importancia, que la capacidad de gestionar el tiempo promueve el éxito académico y que la constancia genera un hábito de un nuevo comportamiento, lo cual contribuye que el estudiante genere estrategias de aprendizaje (Krause y Coates, 2008), por lo cual la gestión de tiempo permite que los estudiantes universitarios continúen con los retos que se presenten en la vida académica y más aún que nos encontramos utilizando aulas virtuales. En este sentido la gestión del tiempo es la expresión conductual de una serie de procesos internos vinculados con la autorregulación para que el individuo tenga probabilidades de alcanzar sus metas, y seguir sus objetivos en la medida que emplee tiempo en realizarlas (Kearns \& Gardiner, 2007).

El estudio tuvo limitaciones ya que se consideró a estudiantes universitarios de una sola universidad de la ciudad de Juliaca, asimismo, por su naturaleza (diseño transversal y alcance correlacional). En este sentido, se recomienda que los próximos estudios sean de carácter experimental o longitudinal; además, sería pertinente realizar investigaciones que analicen el compromiso académico con otras variables como motivación académica, rendimiento académico, estrés académico, autoeficacia.

Se concluye que en la medida que un estudiante universitario asuma la responsabilidad de la gestión de tiempo en su vida académica entonces mayor es el esfuerzo y la energía respecto a lo que el estudiante hace en su vida universitaria.

\section{Correspondencia}

\section{Cahuana Cuti Daniel Eduardo \\ Correo electrónico: \\ danielcahuana@upeu.edu.pe}

\section{REFERENCIAS}

Analytics, P. (2017). En Perú, el $27 \%$ de los ingresantes a universidades privadas abandonan su carrera en el primer año de estudios. https:// www.analytics.cl/educacion/peru-27-losingresantes-universidades-privadas-abandonancarrera-primer-ano-estudios/

Baños, J. (2020). Gestión del tiempo y compromiso académico en estudiantes de psicología de la Universidad Privada Norbert Wiener, $2019 . \quad$ https://repositorio.usmp.edu.pe/ handle/20.500.12727/6828?show=full

Casuso H, M. (2011). Estudio del estrés, engagement y rendimiento académico en estudiantes universitarios de Ciencias de la Salud. Universidad de Málaga, Servicio de Publicaciones. https:// riuma.uma.es/xmlui/handle/10630/4926

Cotrina, M., \& Sanchez, W. (2020). La gestión del tiempo como factor predictor del rendimiento académico de los estudiantes en la Institución Educativa "Dos de Mayo" de Cajamarca 2019. http://repositorio.upagu.edu.pe/handle/ UPAGU/1247

Escurra Mayaute, L. (1988). uantificación de la validez de contenido por criterio de jueces. Revista De Psicología, 6(1-2), 103-111. http://revistas.pucp. edu.pe/index.php/psicologia/article/view/4555

Ferreyra, M., Avitabile, C., Botero, J., Haimovich Paz, F., \& Urzúa, S. (2018). Momento decisivo: La educación superior en América Latina y el Caribe. https://openknowledge.worldbank.org/ bitstream/handle/10986/26489/211014ovSP. pdf? sequence $=5 \&$ isAllowed $=y$

García, R., \& Pérez, F. (2012). Spanish versión of the time management behavior questionnaire for university students (Versión en español del cuestionario de conducta en la gestión del tiempo para estudiantes universitarios). The spanish journal of psychology, 15(3), 1485-1494. doi:10.5209/rev_sjop.2012.v15.n3.39432

Gros, B. (2018). La evolución del e-learning: del aula virtual a la red. Iboamericana de Educación a Distancia, 21(2), 167-191. doi:https://doi. org/10.5944/ried.21.2.20577

Hernández, R., Fernández, C., \& Baptista, P. (2014). Metodologia de la investigación-Sexta edición. https://www.uca.ac.cr/wp-content/ uploads/2017/10/Investigacion.pdf

Hinrichs, C., Ortiz, L., \& Perez, L. (2016). Relación entre el Bienestar Academico de Estudiantes de Kinesiologia de una Universidad Tradicional de Chile y su percepción del Ambiente Educacional. https://scielo.conicyt.cl/scielo.php?script=sci_ 
arttext\&pid=S0718-50062016000100012\&lng $=e$ n\&nrm=iso\&tlng=en

Kearns, H., \& Gardiner, M. (2007). Is it time well spent? The relationship between time management behaviours, perceived effectiveness and workrelated morale and distress in a university context ( ¿Es tiempo bien gastado? La relación entre los comportamientos de gestión del tiempo ). Higher Education Research \& Development. https://doi. org/10.1080/07294360701310839

Krause Lee, K., \& Coates, H. (2008). Students' engagement in first-year university (Participación de los estudiantes en la universidad de primer año). Assessment \& Evaluation in Higher Education. https://doi.org/10.1080/02602930701698892

Macan, T., Shahani, C., Dipboye, R., \& Phillips, A. (1990). College students time management: Correlations with academic performance and stress. ( Gestión del tiempo de los estudiantes universitarios: correlaciones con el rendimiento académico y el estrés). Journal of educational psychology, 82(4), 760-768. doi:10.1037/00220663.82.4.760

Marchena, E., Hervias, F., Galo, C., \& Rapp, C. (2017). Organiza tu tiempo de forma eficaz. 4. https://sap.uca.es/wp-content/uploads/2017/03/ Gu\%C3\%ADa-de-organizaci\%C3\%B3n-deltiempo.pdf?u

Minedu. (2020). Minedu: 174 mil alumnos dejaron sus estudios universitarios este 2020. Canal N. https://canaln.pe/actualidad/coronavirus-174mil-alumnos-dejaron-sus-estudios-universitarioseste-ano-n425757

Moreno N, V. (2019). Propiedades psicométricas de la Escala de Utrecht https://repositorio.upao. edu.pe/bitstream/20.500.12759/4782/1/RE PSICOL_VIVIANA.MORENO_PROPIEDADES. PSICOM\%C3\%89TRICAS_DATOS.PDF

Ordoñez M, D. (2020). Resiliencia y compromiso académico en estudiantes de una universidad de Quevedo, 2020. Universidad Cesar Vallejo Repositorio digital institucional. https://repositorio. ucv.edu.pe/handle/20.500.12692/47952
Organización de las Naciones Unidas. (2020). ONU. Covid-19 y educación superior: Educación y ciencia como vacuna contra la pandemia. Impacto Académico. https://www.un.org/ es/impacto-acad\%C3\%A9mico/covid-19-yeducaci\%C3\%B3n-superior-educaci\%C3\%B3ny-ciencia-como-vacuna-contra-la-pandemia.

Palacio, J., Caballero, C., Gonzales, O., Gravini, M., \& Contreras, K. (2012). Relación del burnout y las estrategias de afrontamiento con el promedio academico. Universitas Psychologica, 11(2), 535544. http://www.scielo.org.co/pdf/rups/v11n2/ v11n2a15.pdf

Parra, P., \& Pérez, C. (2010). Propiedades psicometricas de la escala de comportamiento academico, UWES (versión abreviada), en estudiantes de psicologia. Revista Educativa Ciencias de Salud, 7(2-9, 128-133. http:// www2.udec.cl/ofem/recs/anteriores/vol722010/ artinv7210c.pdf

Schaufeli, W., \& Bakker, A. (2003). Utrecht work engagement scale. Holanda: Utrecht university. https://www.wilmarschaufeli.nl/publications/ Schaufeli/Test\%20Manuals/Test_manual_ UWES_English.pdf

Schaufeli, W., Martínez, A., Marques, A., Salanova, M., \& Bakker, A. (2002). Burnout and engagement in university students: a cross-national study. Journal of Croos (Burnout y engagement en estudiantes universitarios: un estudio transnacional. Diario de la Cruz). Cultural Phychology, 33, 464-481. https://journals.sagepub.com/ doi/10.1177/0022022102033005003

Van L, S., \& Elen, J. (2017). In search of attributes that support self-regulation in blended learning environments. Education and information Technologies, 22(4), 1395-1454.

Recibido: 27/06/2021

Aceptado: 15/10/2021 